\title{
Discours
}

Revue de linguistique, psycholinguistique et

informatique. A journal of linguistics, psycholinguistics

and computational linguistics

$7 \mid 2010$

Varia

\section{Dislocations à gauche et antépositions de compléments en anglais : formes, fonctionnalités et insertion en discours}

\section{Laure Gardelle}

\section{OpenEdition \\ Journals}

Édition électronique

URL : http://journals.openedition.org/discours/7943

DOI : $10.4000 /$ discours. 7943

ISSN : 1963-1723

Éditeur :

Laboratoire LATTICE, Presses universitaires de Caen

\section{Référence électronique}

Laure Gardelle, «Dislocations à gauche et antépositions de compléments en anglais : formes, fonctionnalités et insertion en discours », Discours [En ligne], 7| 2010, mis en ligne le 20 décembre 2010, consulté le 19 avril 2019. URL : http://journals.openedition.org/discours/7943 ; DOI : 10.4000/ discours.7943

\section{(c) (i) $\odot$}

Discours est mis à disposition selon les termes de la licence Creative Commons Attribution - Pas d'Utilisation Commerciale - Pas de Modification 4.0 International. 

Revue de linguistique, psycholinguistique et informatique

Dislocations à gauche et antépositions de compléments en anglais : formes, fonctionnalités et insertion en discours

\author{
Laure Gardelle \\ ENS de Lyon \\ 15 parvis René Descartes \\ BP 7000 \\ 69342 Lyon Cedex o7 \\ laure.gardelle@ens-lyon.fr
}

Laure Gardelle, «Dislocations à gauche et antépositions de compléments en anglais: formes, fonctionnalités et insertion en discours», Discours, 7 | 2010, [En ligne], mis en ligne le 20 décembre 2010. 



\title{
Dislocations à gauche et antépositions de compléments en anglais : formes, fonctionnalités et insertion en discours
}

Laure Gardelle

ENS de Lyon

\begin{abstract}
Parmi les énoncés non canoniques de l'anglais, deux procédés permettent de placer à l'initiale un constituant dont le référent est un participant au procès : la dislocation à gauche et l'antéposition. Certaines études générativistes en ont d'ailleurs conclu qu'elles étaient identiques dans la majeure partie de leur genèse. Pourtant, l'insertion de ces deux structures en discours est contrainte de manière très différente par le cotexte gauche, et elles ne répondent pas non plus aux mêmes fonctionnalités du point de vue de la structure informationnelle. Par exemple, le constituant disloqué ne peut être que topique à l'échelle de la proposition, alors que le constituant antéposé peut également être focus; par ailleurs, l'insertion en discours d'une structure antéposée est plus fortement contrainte par le cotexte gauche, et pourtant, en anglais, cette structure est bien plus fréquente que la dislocation. La présente étude vise à comparer les deux structures, pour préciser leurs fonctionnalités et les contraintes d'emploi, mais aussi pour voir dans quelle mesure la position non canonique du constituant disloqué ou antéposé à l'initiale influe sur ces fonctionnalités. L'analyse invite à conclure que la position initiale a une influence, mais qu'il existe un facteur encore plus important, à savoir la manière dont le constituant placé à l'initiale est rattaché syntaxiquement à la proposition noyau.
\end{abstract}

Mots clés: antéposition, argument, cohésion discursive, dislocation à gauche, insertion en discours, structure informationnelle, topique/focus

Among the non canonical utterances of English, two show in initial position a constituent whose referent is a participant in the event. These two structures are preposing and left dislocation. Some generative studies have concluded that their generation was largely similar. Yet they do not abide by the same constraints in discourse and do not share the same roles in the information structure. For example, a dislocated constituent may only be a topic at clause level, while a preposed element can have either topic or focus status; moreover, the use of a preposing structure is much more constrained by the leftward context, and yet preposing is more common in English than left dislocation. The study seeks to compare the two structures in order to determine their roles and to find out to what extent a non canonical initial location for a dislocated or a preposed constituent influences those roles. It concludes that being in initial position does play a role, but that a more significant factor is the syntactic relation of the dislocated or of the preposed constituent to the nucleus.

Keywords: argument, cohesion, information structure, insertion in discourse, left dislocation, preposing, topic/focus

Parmi les énoncés non canoniques de l'anglais, deux procédés permettent de placer à l'initiale un constituant dont le référent est un participant au procès. Il s'agit de la dislocation à gauche, comme dans «this poor little turtle, it takes him like ten chapters», et de l'antéposition du complément, illustrée par «astronomy I was interested in». Notons que ce dernier mode permet plus largement l'antéposition 
de tout constituant interne au syntagme verbal (SV). On se propose de comparer dislocations à gauche et antépositions d'éléments internes au SV, pour définir leurs fonctionnalités et les conditions de leur insertion en discours, ainsi que pour déterminer si ces caractéristiques sont liées à la forme de chacune des structures.

Les deux types de structures présentent des caractéristiques largement similaires du point de vue de leur forme. Dans l'énoncé de surface, leur principale différence réside dans la présence ou non d'un clitique dans le noyau; et si l'on considère leur genèse, les premières études de grammaire générative les présentent comme le fruit d'une même règle de transformation syntaxique, le mouvement en WH-. Dans son étude fondatrice sur ces phénomènes, Ross (1973: 253) écrit ainsi : «the rule of Topicalization [is] a process which is formally almost identical to Left Dislocation, with the exception that while the latter rule leaves behind a pronoun to mark the position in the sentence that the fronted NP used to occupy, the rule of Topicalization does not» '. Pourtant, l'insertion de ces deux structures en discours n'est pas contrainte de la même manière par le cotexte gauche, et ne répond pas aux mêmes fonctionnalités du point de vue de la structure informationnelle. Quelles sont ces différences fonctionnelles entre les deux structures, et s'expliquent-elles par les quelques différences de forme?

\section{Les dislocations à gauche}

En anglais, la dislocation à gauche est réservée à des énoncés oraux et informels tels que des conversations; aucune occurrence n'a pu être trouvée dans des discours ou dans des interviews d'hommes politiques, par exemple. Même au sein des énoncés informels, elle reste très rare: sur plusieurs dizaines d'heures d'enregistrements de conversations informelles (corpus IViE et $\mathrm{MICASE}^{2}$ ), nous n'avons pu recenser pour la présente étude que II occurrences (voir annexe) ${ }^{3}$. La dislocation à gauche est donc bien plus rare en anglais qu'elle ne l'est en français, par exemple. De Cat (2007: I) constate d'ailleurs: «their pervasive use is one of the distinctive traits of spoken French» ${ }^{4}$ (italiques ajoutés), tandis qu'Agatha Christie fait de l'usage abusif

1. «La règle de Topicalisation [constitue] un procédé quasiment identique, d'un point de vue formel, à la Dislocation à gauche, excepté qu'avec cette dernière, la position qu'occupait le SN antéposé se retrouve marquée par un pronom, ce qui n'est pas le cas avec la règle de Topicalisation» (je traduis). Chez Ross, topicalisation est à entendre au sens plus large d'antéposition.

2. IViE est un corpus oral d'anglais britannique urbain (http://www.phon.ox.ac.uk/files/apps/IViE/search. php); MICASE, ou Michigan Corpus of Academic Spoken English, un corpus oral d'anglais américain utilisé en contexte universitaire (cours, rencontres entre étudiants et tuteurs... : http://micase.elicorpora. info/).

3. Étant donné ce faible nombre, on a choisi d'ajouter les 4 occurrences rencontrées dans des œuvres de fiction, bien que celles-ci ne soient pas authentiques, d'autant plus qu'elles ont confirmé les conclusions tirées des énoncés d'IViE et de MICASE. Les occurrences de dislocation à gauche étant peu nombreuses, elles sont présentées en annexe à la fin du présent article.

4. «Leur utilisation très fréquente constitue l'un des traits distinctifs du français oral» (je traduis). 
de dislocations à gauche un trait linguistique qui trahit l'origine francophone de son détective Hercule Poirot (Leonarduzzi et Herry, 2005: 9). La plupart des grammaires de l'anglais ne mentionnent d'ailleurs pas cette forme de réagencement, en particulier avant les années 2000 - elle est par exemple absente de la grammaire de référence de Quirk et al. (1972), alors qu'elle est traitée trente ans plus tard dans celle de Huddleston et Pullum (2002).

\subsection{Caractéristiques formelles}

La dislocation à gauche se caractérise par la présence à l'initiale d'un constituant, généralement un syntagme nominal $(\mathrm{SN})$, puis d'une proposition dans laquelle se trouve un clitique co-référent. Cette proposition est qualifiée de noyau de l'énoncé. Le clitique peut occuper diverses fonctions syntaxiques, qu'illustrent les énoncés suivants :

[I] (sujet) Blanche, this trip to the Bahamas, it is not with a doctor (série TV, US, $1990^{5}$ )

[2] (complément d'objet) history, I took that in my junior year (MICASE)

[3] (complément d'une préposition) anatomy really, she was talking to us about it one day

(MICASE)

Ces fonctions syntaxiques peuvent être instanciées dans la proposition principale, comme dans les énoncés [I] à [3], mais aussi dans une subordonnée, comme en [4]:

[4] Oh, anything about the office, don't you think you should discuss that with Henry himself?

(M. Binchy)

Il arrive également que deux clitiques aient un même antécédent disloqué, ainsi en $[5]$ :

[5] This guy in my class, I gave bim my notes and he never returned them.

(exemple emprunté à Huddleston et Pullum, 2002: 1409)

Quant au constituant placé à l'initiale, il est qualifié de «disloqué» car il est toujours séparé de la proposition qui suit par une pause. Un constituant intercalé peut même venir accroitre la séparation; ainsi un adverbe de commentaire (énoncé [3] ci-dessus), ou encore, dans les variantes ci-dessous, une proposition de commentaire [6], une interjection [7] ou un vocatif [8]:

[6] this poor little turtle - fancy that (/would you believe it) - it takes him like ten chapters

5. Voir les références complètes des sources, ainsi que ces énoncés avec plus de contexte, en annexe. 
[7] this poor little turtle, man (/gosh), it takes him like ten chapters

[8] this trip to the Bahamas, Blanche, it is not with a doctor

\subsection{Insertion en discours de la dislocation à gauche}

Du point de vue de la structure informationnelle, toutes les études (voir notamment Lambrecht, I994 et Prince, I998) montrent que le constituant disloqué ne peut être que topique, entendu ici comme ce à quoi est rapportée la prédication énoncée dans la proposition noyau. L'énoncé [I], par exemple, peut être glosé ainsi : à propos de ce voyage aux Bahamas, je dis qu'il ne s'effectue pas en compagnie d'un médecin. De même en [2] : à propos du cours d'bistoire, je dis que je l'ai suivi en première année. Un constituant disloqué ne peut être focus, que ce terme soit pris au sens strict d'élément porteur de l'accent nucléaire ou au sens plus large de constituant porteur de la charge informative nouvelle de l'énoncé. Il s'agit là d'une différence avec les antépositions, qu'il s'agira d'expliquer. S'il ne peut être focus, il peut en revanche désigner un référent nouveau pour le coénonciateur, comme en [9], où le référent de my sister Chrissie n'a pas été explicitement posé en amont, bien qu'il soit inclus dans le we qui précède:

[9] We went to Florida last summer, and we went to Disney World. The best ride the whole time was Jurassic Park. My sister Chrissie, her eyes were poppin' out.

(emprunté à Manetta, 2007: IO30)

Cependant, la très grande majorité des constituants disloqués à gauche ont pour référent un élément déjà introduit en amont, comme dans les énoncés [I] à [8].

D’un point de vue pragmatique, la dislocation à gauche peut avoir différentes fonctions, étudiées notamment par Geluykens (I992), Lambrecht (I994), Prince (1997, 1998), Manetta (2007) et Tizón-Couto (2009). Selon les études, le nombre de ces fonctions est variable, mais dans un contexte de comparaison avec les antépositions, elles peuvent être réduites à deux. La plus fréquente est la fonction de simplification, de l'encodage comme du décodage. Elle peut être motivée par divers facteurs. Considérons pour commencer l'énoncé [9], énoncé dans lequel la variante non disloquée [9'] apparaît moins spontanée :

[9'] We went to Florida last summer, and we went to Disney World. The best ride the whole time was Jurassic Park. My sister Chrissie's eyes were poppin' out.

(emprunté à Manetta, 2007: 1030)

Le syntagme nominal my sister Chrissie's eyes exige un effort très important d'encodage et de décodage: le groupe my sister Chrissie introduit le référent, et se trouve dans le même temps enchâssé dans un constituant plus large, dans lequel il sert de déterminant pour l'identification d'un autre référent (les yeux), lui aussi mentionné pour la première fois et non préparé par le cotexte gauche. La dislocation à gauche permet de distinguer les deux opérations: la référence de my sister Chrissie est posée dans un premier temps par le constituant disloqué, puis dans un second 
temps seulement est énoncée une prédication, dans laquelle le rôle du référent est indiqué par le déterminant possessif her. La dislocation permet ainsi de respecter le Principe de séparation du rôle et de la référence (PSRR), principe mis en évidence par Lambrecht (1994: 185) pour les expressions topiques et formulé ainsi : «Do not introduce a referent and talk about it in the same clause» ${ }^{6}$.

Prince (1998: 6) réserve à ces cas de dislocation à gauche avec référent nouveau le terme de «Simplifying Left Dislocation», mais la fonction de simplification nous paraît concerner également des énoncés dans lesquels le référent du constituant disloqué a été introduit en amont. On trouve par exemple:

[ı] Si: right, and we chose [the name] Biotron because we felt it reflected the interaction, the biology of below ground rather than just the focus on roots. $-\mathrm{S}_{2}$ : the one that's up at the biological station and the one at East Ma-Malling, these're the two main ones?

(interview d'un scientifique sur les unités Biotron, MICASE)

Là encore, le constituant disloqué crée une unité informationnelle qui pose la référence. L'effort d'encodage est ensuite moindre par rapport à une structure non disloquée, car la référence est déjà établie au moment où la prédication est énoncée. Le choix d'une opération en deux temps est ici motivé par un changement de topique: après une discussion relative au choix du nom, $\mathrm{S}_{2}$ passe à l'inventaire des stations. La longueur du constituant disloqué contribue peut-être au recours à la dislocation, en ce qu'elle exige déjà en elle-même un effort d'encodage. Mais elle ne constitue pas une condition nécessaire: la variante [ıo'], qui comporte un constituant disloqué plus court, aurait été tout à fait acceptable:

[ı'] Si: right, and we chose [the name] Biotron because we felt it reflected the interaction, the biology of below ground rather than just the focus on roots. - S2: Epping Forest and East Malling, these're the two main ones?

Le fait que le constituant disloqué soit formé de deux GN coordonnés ne constitue pas non plus une condition nécessaire, comme l'ont montré les énoncés [I] à [8]. Considérons l'énoncé $[\mathrm{I}]$, par exemple, reproduit ci-dessous en [II] avec plus de cotexte:

[II] (la locutrice est désespérée par la conduite de sa nièce) SI: And now she's flying off to the Bahamas with a doctor! - S2: Blanche... Blanche, this trip to the Bahamas, it is not with a doctor.

Le recours à la dislocation à gauche s'explique encore par sa fonction de simplification, qui, ici, vise plus spécifiquement le décodage. La répétition de Blanche montre la difficulté qu'éprouve $S_{2}$ à retenir l'attention de $S_{I}$; la dislocation permet de répartir les informations en deux groupes, afin d'en faciliter le traitement

6. «Ne pas introduire un référent et en parler dans une même proposition» (je traduis). 
par un Si en proie à l'émotion. Le constituant disloqué this trip to the Babamas recentre d'abord l'attention de Si sur le référent dont il va être question, puis une prédication est rapportée à ce référent. On note là encore que la dislocation est conditionnée par un changement de topique de discours: un traitement en deux temps deviendrait inacceptable si les propos de $S_{2}$ conservaient pour topique la nièce, comme en [II'] :

[II'] Si: And now she's flying off to the Bahamas with a doctor! - S2: *Blanche... Blanche, your niece, she is not flying to the Babamas.

En effet, il serait alors inutile de recentrer l'attention de Si sur sa nièce hors prédication: il s'agit déjà du topique de discours. Plus généralement, les is occurrences de dislocation à gauche réunies dans le cadre de cette étude, de même que les exemples cités par divers linguistes, présentent toutes un changement de topique de discours. Ce changement de topique s'accompagne parfois d'un effet de contraste; dans ce cas, la dislocation peut devenir obligatoire - phénomène rare et apparemment jamais noté -, ainsi en [12] :

[I2] I - students who say oh well I might as well just take, Honors Math because I need another honors course, those are the students who probably shouldn't be taking Honors Math but the people who like math and they say I like math I and I really enjoy it and and I wanna learn more, they're the people who really should be taking Honors Math

(MICASE)

Les deux pronoms en italiques portent une emphase intonative, pour un effet de contraste entre ceux qui méritent de choisir le cours et les autres. Les deux SN disloqués étant longs et donc porteurs d'une grande charge informative, ils ne pourraient être utilisés dans une phrase canonique pour à la fois introduire le référent et signaler l'emphase. La dislocation, obligatoire, permet une fois encore de séparer référence et rôle.

La seconde grande fonction pragmatique de la dislocation à gauche, isolée par Prince (1998: 6), consiste à instaurer une relation de type Poset - Poset étant l'abréviation de "partially-ordered set», ou ensemble partiellement ordonné7. Cela signifie que le constituant disloqué sert alors à identifier le référent comme membre d'un ensemble d'entités déjà évoqué, avec lequel il entretient une relation saillante, de type membre de ou type de le plus souvent. Ainsi en [13]:

[13] So I see my youngest brother a lot too. Actually, all my brothers are pretty close by. My oldest brother is a chef, like, downtown and my middle brother lives in Jersey. My youngest brother, he's a freshman at Newman.

(exemple emprunté à Manetta, 2007: 1030)

7. Pour plus de détails sur les relations Poset, voir notamment Hirschberg, 1985; Ward, I988 et Cresswell, 2004 . 
[I3'] [...] My oldest brother is a chef, like, downtown and my middle brother lives in Jersey. My youngest brother is a freshman at Newman.

La simplification de l'encodage ou du décodage n'est clairement pas la raison d'être de la structure disloquée dans cet exemple. Pour Manetta, la dislocation à gauche en [13] vise à relier plus explicitement le référent de my youngest brother à l'ensemble des/frères de la locutrice/. Si l'on compare l'énoncé à sa variante canonique [I3'], il nous semble que le recours à la dislocation, parce qu'il brise le parallélisme des prédications de type «tel frère fait telle chose », permet également de ne pas traiter ce plus jeune frère simplement comme l'un des membres de l'ensemble, mais de recentrer plus fortement le discours sur lui dans la dernière phrase, à l'image de la toute première phrase de l'extrait.

En conclusion, les deux grandes fonctions pragmatiques de la structure s'expliquent par un procédé unique : dans tous les cas, il s'agit de séparer la fonction de référence du SN, donnée par le constituant disloqué, et le rôle d'argument du référent dans le procès, rôle précisé par le clitique. L'information est alors traitée en deux étapes, l'élément disloqué constituant une unité de traitement informationnel. Ce traitement en deux temps, en anglais, doit s'accompagner d'un changement de topique, à l'échelle du discours ou de la proposition, pour être grammaticalement acceptable. La dislocation à gauche est obligatoire dans les très rares cas où le constituant disloqué est long et le clitique co-référent porteur d'emphase; ailleurs, le recours à la dislocation repose sur un choix énonciatif, et il est considéré comme la marque d'un registre très oral et informel.

\subsection{Un lien entre forme et fonctionnalités?}

Il s'agit à présent de déterminer si les caractéristiques syntaxiques de la dislocation à gauche (place du constituant disloqué à l'initiale, pause, reprise par un clitique dans la proposition noyau) expliquent les propriétés notées, à savoir: le fait que le constituant disloqué ne puisse pas être focus, le fait qu'il puisse introduire un référent nouveau mais qu'il s'agisse le plus souvent de référents déjà établis, le fait qu'il soit repris par un clitique, la séparation du rôle et de la référence opérée par la dislocation, ainsi que les deux fonctions pragmatiques de la structure (simplification du traitement de l'information et relation de type Poset).

Pour répondre, il est nécessaire de s'interroger d'abord sur la nature du rattachement syntaxique du constituant disloqué à la proposition noyau. Notamment, si c'est la forme de la construction qui permet la séparation du rôle et de la référence, le constituant disloqué ne doit pas faire partie intégrante de la prédication et ne doit donc pas occuper une position d'argument.

Dans l'analyse originelle par mouvement en WH-, proposée initialement par Ross (1973: 253), le constituant disloqué constitue un argument de la proposition noyau. Pour Ross, la structure profonde d'un énoncé avec dislocation à gauche est identique à celle de la variante canonique; par exemple, dans l'énoncé [I], la structure profonde ferait apparaittre, de manière très simplifiée, la structure canonique this 
trip to the Babamas - be - with a doctor. Puis après transformation négative, elle subirait un mouvement en $\mathrm{WH}-$, mouvement qui consiste à antéposer le $\mathrm{SN}$ et à lui substituer le clitique $i t$. C'est seulement lors de ce mouvement en WH- qu'aurait lieu l'opération de séparation du rôle et de la référence. Cependant, considérer ainsi la dislocation comme une transformation de mouvement et de copiage pose problème. D'une part, lorsque le clitique est complément d'une préposition, cette dernière ne se retrouve pas à l'initiale du constituant disloqué; ainsi, en [3], on lit "anatomy really, she was talking to us about it one day», et non "*about anatomy really, she was talking to us about it one day». En surface, le constituant disloqué ne présente donc aucun rattachement syntaxique au verbe de la proposition; Larsson (1979: 122), par exemple, en conclut qu'il n'y a pas transformation. De même, dans les très rares cas où le constituant disloqué est un pronom, tel «Me, I...», le pronom disloqué prend la forme oblique, même lorsque le pronom clitique $(I)$ co-référent présente la forme marquée sujet; cette caractéristique suggère que ce pronom disloqué n'occupe pas de fonction syntaxique d'argument. Par ailleurs, la genèse de la dislocation à gauche semble devoir être identique à celle d'énoncés apparentés, qui présentent un constituant isolé à l'initiale et un segment co-référent dans la proposition qui suit, tels que [I4]:

[I4] That brother of yours, the guy's a crook.

(exemple emprunté à Huddleston et Pullum, 2002: I4I2)

Or, dans ce type d'énoncé, les deux constituants étant des SN développés, il est impossible de postuler un mouvement en WH-. Hirschbüler (1974) et Huddleston et Pullum (2002) en concluent que la dislocation n'est pas le résultat d'un mouvement en WH-. Il en va de même pour les constructions à topique détaché, qui, sans être des cas de dislocation, présentent elles aussi un constituant détaché à gauche, comme en $[\mathrm{I} 5]$ :

[I5] The typical family today, the husband and the wife both work.

(exemple emprunté à Lambrecht, 1994: 193)

Dans un tel énoncé, il est impossible de réintégrer the typical family today au sein de la proposition qui suit, et donc de postuler un rattachement syntaxique dans la structure profonde (Lambrecht, 1994: 193).

Face à ces objections, de nombreux générativistes, en particulier depuis les années 1990, rejettent l'analyse de Ross pour proposer une génération du constituant disloqué dans sa position pré-noyau dès la structure profonde, selon différents modèles - voir notamment Cinque (I990), Rizzi (1997) ainsi que le panorama proposé par De Cat (2007: 98-108) et Blasco-Dulbecco (1999: 37-52). Dans ces analyses, le constituant disloqué est généré par une branche spécifique à la gauche de la proposition, branche «Topique» pour certains linguistes (Rizzi, 1997; Frascarelli, 2002), branche sans fonction discursive spécifiée pour d'autres (Cinque, 1990). Le constituant disloqué ne fait à aucun moment partie du noyau de la proposition et n'y occupe jamais de fonction d'argument. 


\subsection{Genèse et caractéristiques formelles}

L'antéposition consiste à placer à l'initiale d'une proposition un constituant interne au SV. Il peut s'agir d'un SN, comme dans les dislocations à gauche; le constituant antéposé est alors le plus souvent complément d'une préposition (ainsi dans «Astronomy I was interested in») ou complément d'objet direct, comme en [I6] : 
[16] Bingley she had likewise seen for an instant.

(J. Austen)

Notons qu'il ne peut y avoir mouvement d'antéposition pour un sujet, celui-ci occupant déjà, dans l'ordre canonique, la position initiale dans la proposition noyau. La gamme de natures et de fonctions syntaxiques des éléments antéposables est plus large que pour les dislocations: il est possible d'antéposer un attribut du sujet [17] ou la partie lexicale d'un verbe et ses compléments [I8] :

[17] That old lady's been on the phone, the one as was down here a few years ago. Bright she is, isn't she?

(A. Christie)

[18] "Help me back, boys," stammered Jack, and belp bim they did. (exemple emprunté à Huddleston et Pullum, 2002)

Du point de vue de la genèse, tous les linguistes s'accordent à dire que l'antéposition résulte d'un mouvement syntaxique en WH-, à partir d'une structure profonde identique à celle qui générerait la phrase canonique. Par exemple, "astronomy I was interested in» partage la même structure profonde que «I was interested in astronomy». À la différence des dislocations à gauche, lors du mouvement syntaxique d'antéposition, la place laissée vide n'est pas instanciée par un pronom, et le constituant déplacé n'est pas isolé du reste de la proposition. C'est pourquoi, au moins lorsque ce constituant est court, toute pause est impossible, de même que l'insertion d'une interjection ou d'un adverbe: *astronomy // I was interested in (où // signale une pause), *astronomy, boy, I was interested in, *astronomy, really, I was interested in.

La distribution en discours des antépositions, enfin, est plus large que celle des dislocations à gauche: elles se rencontrent non seulement dans des conversations informelles, mais aussi dans de l'oral plus formel et à l'écrit, notamment dans les passages narratifs de grands auteurs tels que Charles Dickens ou Jane Austen (voir la sélection d'occurrences du corpus en annexe). Aucune différence n'a pu être notée, du point de vue de l'insertion en discours ou de la fonctionnalité, entre emplois écrits et oraux.

\subsection{Insertion en discours des antépositions}

Du point de vue de la structure informationnelle, toutes les études sur les antépositions (voir notamment Prince, 198I; Ward, 1988; Lambrecht, I994 ou encore Huddleston et Pullum, 2002) ont montré que le complément antéposé pouvait avoir deux statuts diamétralement opposés à l'échelle de la proposition: topique ou focus - à la différence du constituant disloqué à gauche, qui ne peut être que topique. Ces deux statuts sont illustrés par les énoncés suivants:

[19] I did a lot better in the second test. The first test I failed, the second test I got. (littéralement: «J'ai fait bien mieux dans le second devoir. Le premier devoir j’ai raté, le second devoir j'ai réussi.»)

(MICASE) 
[20] The list has included... Kate Stimpson, uh Katherine Stimpson some of you know her as, former dean... (littéralement: «La liste comptait notamment... Kate Stimpson, euh Katherine Stimpson certains d'entre vous la connaissent comme [c'est-à-dire, sous le nom de], ancienne doyenne...»)

(MICASE)

L'énoncé [19] présente deux cas d'antéposition du topique, encore appelés topicalisations. En effet, l'énoncé est glosable par: à propos du premier devoir, je dis que je l'ai raté; à propos du second, je dis que je l'ai réussi. On note que le focus, signalé par l'accent nucléaire, est alors porté par le prédicat (failed et got respectivement). En revanche, l'énoncé [20] n'est pas glosable par: *à propos de Kate Stimpson, je dis que certains d'entre vous la connaissent sous le nom de. C'est le SN Katherine Stimpson qui porte l'accent nucléaire. Il constitue en effet la seule information nouvelle: il s'agit ici de reprendre le signifiant Kate Stimpson pour lui apporter une correction (Katherine Stimpson). [20] illustre donc un cas d'antéposition du focus. Précisons deux restrictions : lorsque le constituant antéposé est attribut, il est toujours focus, tandis qu'à l'inverse, s'il s'agit de la partie lexicale d'un verbe accompagnée de compléments, il ne peut être que topique.

Si le rôle des éléments antéposés dans la structure informationnelle est plus varié que celui des constituants disloqués, l'insertion en discours des antépositions, en revanche, est plus contrainte que celle des dislocations. Prince puis Ward (Prince, I98I ; Ward, 1988) ont montré que le recours à l'antéposition devait respecter deux principes. L'un concerne le constituant antéposé: son référent doit avoir été déjà mentionné ou faire partie de manière saillante d'un ensemble déjà mentionné. De ce fait, le constituant antéposé est nommé lien par les deux linguistes («link»), et l'élément pertinent du cotexte gauche déclencheur («trigger»); ainsi, en [20], Katherine Stimpson est le lien déclenché par Kate Stimpson. Lien et déclencheur doivent tous deux faire partie d'un même ensemble - ici l'ensemble des noms d'usage de Kate Stimpson -, nommé ensemble d'ancrage («anchoring set»). C'est d'ailleurs parce que le lien avec le cotexte gauche s'effectue par l'intermédiaire de cet ensemble d'ancrage que la structure n'exclut pas l'apport d'informations nouvelles dans le SN antéposé, et donc, comme en [20] par exemple, le statut de focus de celui-ci à l'échelle de la proposition.

La seconde contrainte à l'antéposition concerne la proposition dans son ensemble: de cette proposition doit pouvoir être dégagée une prédication ouverte («open proposition»), à une ou plusieurs variables, et cette même prédication ouverte doit pouvoir être inférée du cotexte gauche. Une prédication ouverte ne correspond pas à la relation prédicative effective de l'énoncé, mais consiste en un contenu propositionnel inférable. Ainsi, si l'on considère l'énoncé [19]: de la proposition «the first test I failed» peut être extraite la prédication ouverte [I did X in the first test], où $\mathrm{X}$ est un résultat. L'antéposition n'est acceptable que parce que l'on peut déduire du cotexte gauche cette même prédication: «I did a lot better in the second test» évoque notamment [I did Y (defined as better than X) in the second test], qui 
inclut [I did X in the first test]. C'est pourquoi, dans la proposition à constituant antéposé, failed - qui instancie $\mathrm{X}$ - aurait pu être remplacé par toute remarque liée à des performances ou résultats, ainsi «the first test I did not take very seriously» ou «the first test I was more happy about». Au contraire, l'antéposition aurait été inacceptable avec par exemple: «the first test everybody failed», car la prédication ouverte correspondant à cette proposition aurait fait apparaittre everybody et non plus $I$. Il en va de même lorsque le constituant antéposé est en focus. Ainsi, en [20], la simple mention d'un nom («Kate Stimpson») implique la prédication ouverte [X knows her as $\mathrm{Y}]$, où $\mathrm{X}$ est entendu par défaut comme étant tout le monde; cette prédication ouverte est encore saillante dans la proposition qui présente l'antéposition, puisque l'on peut facilement en inférer [X knows her as $\mathrm{Y}]{ }^{8}$.

Enfin, d'un point de vue pragmatique, Ward (2008:5) a montré que l'antéposition avait une fonction unique : renforcer la cohésion discursive, en établissant un lien avec le cotexte gauche par l'intermédiaire de l'ensemble d'ancrage. Cette conclusion est confirmée par les analyses menées pour la présente étude. Ainsi, si l'on compare l'énoncé [I9] à sa variante canonique [I9'] :

[19] I did a lot better in the second test. The first test I failed, the second test I got.

[19'] I did a lot better in the second test. I failed the first test, I got the second test.

La version canonique parait peu spontanée: il manque un mot de liaison, tel que whereas. On en conclut que c'est le constituant antéposé qui, de par sa position à l'initiale, joue ce rôle de liaison. En effet, parce qu'il est énoncé en premier, il constitue le point de départ de l'énonciation à l'échelle de la proposition. C'est plus précisément à lui qu'est rapportée la prédication qui suit; en ce sens, il acquiert le statut de topique à l'échelle de la proposition, tandis que le sujet $I$ est réduit au rang de simple argument de fail. Dans la variante canonique, c'est $I$ qui aurait constitué le topique, ce statut étant prototypiquement instancié par le constituant sujet. Si l'énonciateur choisit de faire de l'objet le point de départ de l'énonciation, plutôt

8. Précisons que lorsque l'élément antéposé est la partie lexicale du verbe et ses compléments, une contrainte supplémentaire s'ajoute: le verbe doit être identique au verbe déclencheur du cotexte gauche. Ainsi, en [I8], Help me back, boys impose une reprise par belp, à l'exclusion de tout synonyme - *and give bim $a$ hand they did, * and come to the rescue they did. Cette contrainte supplémentaire pourrait s'expliquer par la centralité du verbe dans la prédication: le déplacer constitue un effort d'encodage et de décodage majeur, ce qui pourrait expliquer qu'il ne puisse s'accompagner dans le même temps d'un déplacement sémantique, même léger. À cela pourrait s'ajouter un autre facteur, lié à la fonction pragmatique de l'antéposition d'un élément verbal. Celle-ci est plus spécifique que pour les autres formes d'antéposition: il s'agit toujours de reprendre pour confirmer (comme en [I8]), de suspendre (dans une subordonnée hypothétique, ainsi en [i] ci-dessous) ou d'infirmer (à l'aide d'une négation, ainsi en [ii]) (Huddleston et Pullum, 2002: 1377):

[i] It is odd that Diana should have said that, if sayit she did.

[ii] "Help me back, boys!" stammered Jack. But help him they would not.

En d'autres termes, dans tous ces cas, ce qui importe est la polarité de la prédication, information attachée à l'auxiliaire (did/would not). Cette concentration sur l'auxiliaire pourrait renforcer le caractère inacceptable d'interventions sur la composante lexicale du verbe. 
que de choisir le sujet, c'est parce qu'il existe une raison à l'échelle du discours, au-delà de la seule proposition: il s'agit de renforcer la cohésion avec ce qui précède. Ce principe de cohésion renforcée vaut également lorsque le constituant antéposé est focus. Ainsi, si l'on compare [20] et [20']:

[20] The list has included... Kate Stimpson, uh Katherine Stimpson some of you know her as, former dean...

[20'] The list has included... Kate Stimpson, uh some of you know her as Katherine Stimpson, former dean...

Il n'est plus question de changement de topique, mais là encore, l'énonciateur, en donnant comme première information de la proposition le nom corrigé, lui accorde une importance accrue par rapport à la variante canonique : il donne immédiatement la charge informative nouvelle de la proposition. Cette modification ne peut être motivée discursivement que par le cotexte gauche; ici, l'énonciateur cherche à se corriger immédiatement. Par conséquent, l'énoncé [19] est l'indice d'une réaction immédiate et spontanée, tandis qu'en [19'], la formation canonique suggère une plus grande prise de recul de l'énonciateur, plus de sang-froid dans cette correction, précisément parce qu'il conserve l'ordre canonique, indépendamment de ce qui a été énoncé en amont.

\subsection{Un lien entre forme et fonctionnalité?}

Comme dans le cas des dislocations à gauche, un lien fort apparaît entre les caractéristiques syntaxiques des antépositions d'éléments internes au SV et leurs fonctionnalités. Là encore, deux éléments sont à prendre en compte : la position à l'initiale, mais aussi et surtout la nature du rattachement syntaxique du constituant déplacé à la proposition noyau.

La position à l'initiale place le constituant antéposé au plus près du cotexte gauche, ce qui parait particulièrement en adéquation avec le rôle de renfort de la cohésion discursive que joue la structure. Ceci dit, la position à l'initiale ne suffit pas à expliquer les caractéristiques fonctionnelles de l'antéposition, plus précisément les différences d'emploi par rapport à la dislocation à gauche, qui présente elle aussi un constituant à l'initiale. Il s'agit notamment d'expliquer trois phénomènes. D'une part, pourquoi l'élément antéposé peut être topique, mais aussi focus, alors que le constituant disloqué doit être topique; d'autre part, pourquoi le rôle de renfort de la cohésion discursive constitue la seule motivation de l'opération d'antéposition, avec une continuité forte assurée via l'ensemble d'ancrage, alors qu'il n'est pas aussi central pour les constituants disloqués; enfin, pourquoi aucune pause ou insertion de constituant n'est possible dans le cas de l'antéposition. On propose que ces différentes caractéristiques s'expliquent par le mode de rattachement du constituant antéposé à la proposition noyau.

En effet, à la différence de la construction disloquée, l'antéposition conserve au constituant déplacé la fonction syntaxique de complément interne au SV de la proposition noyau. Il fait donc partie intégrante de la prédication, ce qui explique qu'il 
puisse être porteur du focus ${ }^{9}$ et qu'il ne puisse être isolé du reste de la prédication par une pause. Par ailleurs, les contraintes majeures d'insertion en contexte (prédication ouverte, ensemble d'ancrage) s'expliquent au moins en grande partie par le fait qu'il s'agit du mouvement d'un argument, voire d'une partie du groupe verbal lui-même. L'anglais est en effet une langue dans laquelle l'ordre SVO est fortement prégnant et assez rigide; par exemple, le complément d'objet est très rarement séparé du verbe par un adverbe ou un autre constituant. Par conséquent, le déplacement d'un élément interne au SV vers la position initiale exige une motivation forte, extra-clausale; le fait que le déplacement ait lieu vers l'initiale matérialise l'attraction forte du cotexte gauche, et donc le rôle de renfort de la cohésion discursive.

Une dernière différence entre dislocations à gauche et antépositions concerne leur degré d'acceptabilité en anglais: on a noté que l'antéposition était employée dans tous les registres de langue, tandis que la dislocation à gauche était réservée à un discours oral informel. Il est possible que cette différence soit seulement le fruit du hasard dans l'exploitation de ces deux structures par la langue. Il est possible également que la plus grande acceptabilité de l'antéposition soit liée au fait qu'il s'agit d'un simple déplacement de constituant au sein d'une même proposition noyau, alors qu'avec les structures disloquées, un constituant se trouve en plus isolé hors prédication. Si ce critère avait une influence, il ne suffirait cependant pas à expliquer les différences de degré d'acceptabilité: dans d'autres langues, c'est au contraire l'antéposition qui est beaucoup moins employée que la dislocation à gauche - en français par exemple, elle n'est employée que dans un registre oral spontané avec un SN antéposé focus, ainsi dans un énoncé tel que «plus de Blédine, toute la rangée ils nous ont piqué» (Ghioldi, cité par Blanche-Benveniste, 1997: II4). Le critère évoqué influerait donc en association avec un autre facteur, qui reste à trouver.

\section{Conclusion}

Au terme de cette étude, il apparaît que dislocations à gauche et antépositions ne doivent pas être classées comme deux structures fondamentalement très proches, voire issues d'un même mécanisme. Bien qu'elles aient en commun le placement à l'initiale d'un constituant dont le référent joue un rôle d'argument dans le procès, d'un point de vue syntaxique, pragmatique, informationnel et discursif, elles répondent à des contraintes et à des logiques différentes. L'étude a en effet montré que la seule position initiale, même si elle avait une raison d'être importante, ne suffisait pas à assurer une fonctionnalité : les divergences entre les deux constructions sont liées en grande partie aux modes de rattachement syntaxique des constituants de gauche

9. Ce rattachement explique d'ailleurs deux contraintes supplémentaires notées plus haut. D’une part, la restriction au statut de focus de l'attribut du sujet antéposé : elle est très probablement liée au statut rhématique de ce constituant dans toute phrase. Inversement, on a noté le caractère topical du verbe lexical accompagné de ses compléments en position antéposée; il s'explique par le caractère nécessairement thématique de ces éléments dans un tel contexte de discours. 
à la proposition noyau. L'antéposition déplace à l'initiale un constituant interne au noyau, tandis qu'avec la dislocation à gauche, un constituant est placé hors noyau pour être introduit hors prédication. La position du disloqué à gauche s'explique alors par une volonté de donner la référence avant le rôle. Une comparaison avec la dislocation à droite permettrait de préciser les raisons d'un tel ordre.

\section{Annexe: corpus}

\section{Corpus de dislocations à gauche}

\section{*Énoncés authentiques:}

I. Si: so they've gotta come up with really fantastical ideas like you know all the Silk Cuts

S2: hm

SI: things

S2: yeah the Silk Cuts they're quite funny

(IViE)

2. Si: Biotron was the, the name that's in the literature for similar facilities is Rhizotron.

S2: right, that that's an older name right?

Si: right and we chose Biotron, because we felt it reflected the interaction, the biology of below ground rather than just the focus on roots...

S2: the one that's up at the biological station and the one at East Ma-Malling, these're the two main ones?

SI: no there there're a bunch [S2: uhuh] now

(MICASE)

3. S2: it d- doesn't matter. just wondered whether

$<P A U S E: 06>$ Si: $<L A U G H>$ I have a terrible memory

S2: when you're the second author of a paper that the_ this means that... you're not the primary writer right but you co-author it or you argue about it?

(MICASE)

$4 / 5$. Si: mm. if you like math,

$\mathrm{S}_{3}$ : I do feel, yeah

SI: see I_ students who say ob well I might as well just take, Honors Math because I need another honors course, those are the students who probably shouldn't be taking Honors Math. $\left[\mathrm{S}_{3}: \mathrm{mhm}\right]$ but the people who like math and $\left[\mathrm{S}_{3}: \mathrm{yes}\right]$ they say I like math $I\left[\mathrm{~S}_{3}: \mathrm{I} \mathrm{do}\right]$ and I really enjoy it $\left[\mathrm{S}_{3}: \mathrm{mhm}\right]$ and and I wanna learn more, they're the people who really should be taking Honors Math. [ $\mathrm{S}_{3}$ : right $]$ so maybe you should take the one-eighty-five one-eighty-six you realize that you're going to lose the A-P credit, if you take one-eighty-five and one-eighty-six. $\left[\mathrm{S}_{3}: \mathrm{mhm}\right]$ but, four credits, aren't going to make you or break you.

(MICASE) 
6. Si: did you take A-P Biology?

$\mathrm{S}_{3}$ : no [Sı: no] we didn't have an A-P Bio class, that was... [S I: it's okay] I almost wanna say like, well no actually it's not the same thing my, my anatomy teacher was also the biology teacher [Si: $\mathrm{mhm}$ ] and, anatomy really, she was talking to us about it one day [SI: $\mathrm{mhm}$ ] and, I I really can't say that I did 1- anything like A-P Biology either so, I mean it tries to continue but it it goes through the kingdoms [SI: $\mathrm{mhm}$ ] so, like to the different phylum like the animal, [SI: $\mathrm{mhm}$ ] kingdom so I really don't think

(MICASE)

7. Si: a year of organi- well, this is a semester of inorganic right here now, one onethirty

$\mathrm{S}_{3}$ : oh really?

Si: $\mathrm{mhm}$

$\mathrm{S}_{3}$ : oh

SI: and then organic would be two-ten two-eleven that's one semester and then two-fifteen two-sixteen that's the second semester. and you'd be taking two-fifteen two-sixteen in the fall term next year.

(MICASE)

8. Si: oh you took a lot. now did you take tests in all of those?

$\mathrm{S}_{4}$ : just the lit and history.

Si: okay. and, do you know your scores yet?

$\mathrm{S}_{4}$ : bistory, I took that my junior year and I got a four on that, I haven't gotten the scores

SI: is that the American History?

$\mathrm{S}_{4}$ : yes

(MICASE)

9. $\mathrm{S}_{4}$ : so what does that do for me applying with sophomore, standing?

Si: it means that you get an earlier CRISP date, and so you uh, some of the courses that are typically sort of first-year-student courses, [S4: right] you get a first whack at the them, [ $\mathrm{S}_{4}$ : okay] before some other first-year students will. [ $\mathrm{S}_{4}$ : uhuh] so, you know that's really pretty good. [ $\left.\mathrm{S}_{4}: \mathrm{mhm}\right]$ yeah. so, you want to do sort of a pre-med curriculum or just a free_a pre-biology curriculum or how how have you thought about it?

(MICASE)

Io. $\mathrm{S}_{2}$ : $<L A U G H>$ but that_ you know what I'm saying [S4: yeah] I mean a lot of it of course if you get a $\mathrm{C}$-minus in Math one-oh-five and then you get a $\mathrm{C}$ in Math onefifteen and a $\mathrm{C}$ in econ the Business School's pretty much a wipeout but [S4: right] I've actually asked the Business School advisor this before cuz students will say they wanna withdraw from a course cuz they're gonna get a C. and the Business School would rather see somebody like struggle with it, get the $\mathrm{C}$, move on with their life, do something interesting and challenging the next semester, than withdraw. $\left[\mathrm{S}_{4}\right.$ : 
right] you know I think if you're failing if you have a thirty-three percent overall and you need to get you know a hundred on the next, $\left[\mathrm{S}_{4}: \mathrm{mhm}\right]$ all the next homeworks they're not even collecting them but the next exam in order to get a $\mathrm{C}$-minus then you're being kind of unrealistic because that's not gonna happen.

(MICASE)

II. S2: um and with the Grapes of Wrath there's, you know a lot of Christ imagery as well um, and one of the nice things about that book is it's a fair- it's fairly obvious imagery they kinda, hit you over the head with it I remember the turtle crossing the road there $\left[\mathrm{S}_{4}: \mathrm{mhm}\right]$ I haven't you know I haven't read that book since I was like in tenth grade or something $\left[\mathrm{S}_{4}: \mathrm{mhm}\right]$ but that poor little turtle it takes him like ten chapters to you know how is that like the Joads you know that struggle that kind $\left[\mathrm{S}_{4}\right.$ : uhuh] of stuff.

(MICASE)

* Euvres de fiction:

I2. Si: And now she's flying off to the Bahamas with a doctor! S2: Blanche, this trip to the Babamas, it is not with a doctor. (série TV «The Golden Girls», US, 1990)

I3. "-and if they'd been on bad terms lately-" / But Miss Marple interrupted him. "Oh, but they hadn't." / "You know that for a fact?" / "Everyone would have known if they'd quarrelled! The maid, Gladys Brent - she'd have soon spread it round the village." [les barres obliques indiquent un changement de paragraphe]

(A. Christie, Miss Marple's Final Cases, Londres, Harper, 1960)

I4. "You see, one of Miss Emily's brooches was missing, and such a hue and cry for it as never was [...]. And Gladdie's helped search everywhere, and there was Miss Lavinia saying she was going to the police about it, and then it turned up again, pushed right to the back of a drawer in the dressing-table, and very thankful Gladdie was. / And the very next day as ever was a plate got broken, and Miss Lavinia she bounced out right away and told Gladdie to take a month's notice."

(A. Christie, Miss Marple's Final Cases)

I5. Then he said, "I'm rather worried about Henry at the office, this is really what I wanted to talk to you about." / Every antenna was quivering. She did not want to hear this conversation, she did not like the way it was being done. "Oh, anything about the office, don't you think you should discuss that with Henry himself?"

(M. Binchy, Light a penny candle, New York, Penguin, 1982)

I6. "Will we have a joint birthday party?" / "That's a great idea. I'd like that. Any excuse is good enough for me." / "Right, will we have it in your place? It's bigger." / "No, the neigbbours, they're difficult, and the stairs."

(M. Binchy, Light a penny candle) 


\section{Corpus d'antépositions: sélection}

\section{*Énoncés authentiques:}

I. Si: I think educationally it would be better for you to struggle through it [the course] than to run away with your tail between your legs.

S2: well no because I mean I don't, cuz the problem is is like you know and then I gotta take again next semester and it's just, [SI: right] yeah.

SI: right

S2: I mean, like I mean cuz I did a lot better on the second test the first test I failed the second test $\mathrm{i}$ got, like (i-) if you took it just ouf of like a raw where it_ no curve I did id did you know I did bad, but because there was a curve and everybody else did bad I didn't do as bad

(MICASE)

2. S2: biology?

SI: nope nope I hate biology I never did like it

S2: bio- th- th- then that should not be _ there's a lot of sciences maybe you could take astronomy or

SI: astronomy I was interested in maybe I'll take that some time

(MICASE)

3. and the thing is it_ wi- with these uh, with organizational studies I will have like, if I wanna like_summer I don't wanna spend in Michigan. it's like um it's like, I don't know maybe travel the country and s- go to other universities in the big cities to study?

\section{(MICASE)}

4. the list has included uh not only Larry Levine who is (a historian now) at George Mason University and Edward Said he's at Columbia, but as recently as last week, uh Kate Stimpson Katherine Stimpson some of you know her as, former dean of the graduate school of (Rutgers) she's now dean of the graduate school at N-Y-U, and in between she was at the McArthur Foundation. um the list also in- includes [...]. (MICASE, discours public d'un doyen)

\section{* Euvres de fiction:}

5. "Just wanted to... well... what poor William said and all that, you know?" / "He's been abroad a long time," said Mrs Eccles, "and I think he must have had some very nasty experiences. Very quiet and depressed he's been, ever since he came home." (A. Christie, Miss Marple's Final Cases)

6. "He won't get too far, ma'am," he said. "That's to say he won't get far unobserved, if you take my meaning." / "Oh," said Bunch, relieved. / "That old lady's been on the phone," said Police Constable Abel, "the one as was down here a few years ago. Bright she is, isn't she?"

(A. Christie, Miss Marple's Final Cases) 
7. Elizabeth said as little to either [Darcy and Bingley] as civility would allow, and sat down again to her work, with an eagerness which it did not often command. She had ventured only one glance at Darcy. He looked serious, as usual; and, she thought, more as he had been used to look in Hertfordshire, than as she had seen him at Pemberley. But, perhaps he could not in her mother's presence be what he was before her uncle and aunt. It was a painful, but not an improbable, conjecture. Bingley she had likewise seen for an instant, and in that short period saw him looking both pleased and embarrassed.

(J. Austen, Pride and Prejudice [1813], Ware (Hertfordshire), Wordsworth, I992)

8. On the present occasion, though I was hungry, I dared not eat my slice. I felt that I must have something in reserve for my dreadful acquaintance, and his ally the still more dreadful young man. I knew Mrs Joe's housekeeping to be of the strictest kind, and that my larcenous researches might find nothing available in the safe. Therefore I resolved to put my hunk of bread-and-butter down the leg of my trousers.

The effort of resolution necessary to the achievement of this purpose, I found to be quite awful. It was as if I had to make up my mind to leap from the top of a high house, or plunge into a great depth of water.

(Note: la virgule qui suit le constituant antéposé ici ne signale pas une pause; elle est due à la longueur du constituant.)

(C. Dickens, Great Expectations [I86I], New York - Londres, Norton, I999)

\section{Bibliographie}

Blanche-Benveniste, C. 1997. Approches de la langue parlée en français. Paris: Ophrys.

Blasco-Dulbecco, M. 1999. Les dislocations en français contemporain: étude syntaxique. Paris: Champion.

Cinque, G. 1990. Types of $\bar{A}-$ Dependencies. Cambridge (Mass.) : MIT Press.

CResswell, C. 2004. Syntactic Form and Discourse Function in Natural Language Generation. New York: Routledge.

De Cat, C. 2007. French Dislocation: Interpretation, Syntax, Acquisition. Oxford: Oxford University Press.

Frascarelli, M. 2002. Topicalization as Merge. A Comparative Analysis of Left and Right Dislocation in Italian. Manuscrit. Università degli Studi Roma Tre.

Geluykens, R. 1992. From Discourse Process to Grammatical Construction. On Left-Dislocation in English. Amsterdam - Philadelphia: John Benjamins.

Givón, T. 20or. Syntax: an introduction. Amsterdam - Philadelphia: John Benjamins. Vol. 2.

Hirschberg, J. 1985. A Theory of Scalar Implicature. $\mathrm{PhD}$ thesis. Department of Linguistics, University of Pennsylvania.

HiRsChbüler, P. 1974. La dislocation à gauche comme construction basique du français. In C. Rohrer et N. Ruwet (eds), Actes du colloque franco-allemand de grammaire transformationnelle. Tübingen: M. Niemeyer. Vol. I: Études de syntaxe: 9-17. 
Huddleston, R. et Pullum, G.K. 2002. The Cambridge Grammar of the English Language. Cambridge: Cambridge University Press.

Lambrecht, K. 1994. Information Structure and Sentence Form: Topic, Focus, and the Mental Representation of Discourse Referents. Cambridge: Cambridge University Press.

LARSSON, E. 1979. La dislocation en français. Étude de syntaxe générative. Lund: CWK Gleerup.

Leonarduzzi, L. et Herry, N. 2005. Les dislocations: textes et contextes. Congrès de la Société des anglicistes de l'enseignement supérieur, Toulouse. Accessible en ligne sur http://hal.archives-ouvertes.fr/docs/oo/48/42/04/PDF/430o.pdf (consulté le 20/or/IO).

Manetta, E. 2007. Unexpected Left Dislocation: an English Corpus Study. Journal of Pragmatics Vol. 39: I029-I035.

PrInCE, E. 198I. Topicalization, Focus-Movement, and Yiddish-Movement: A Pragmatic Differentiation. In D. Alford et al. (eds), Proceedings of the Seventh Annual Meeting of the Berkeley Linguistics Society. Berkeley: Berkeley Linguistics Society, University of California: 249-64.

PrInCE, E. 1997. On the Functions of Left-Dislocation in English Discourse. In A. Kamio (ed.), Directions in Functional Linguistics. Amsterdam - Philadelphia: John Benjamins : II7-I44.

Prince, E. 1998. On the Limits of Syntax, with Reference to Left-Dislocation and Topicalization. Syntax and Semantics Vol. 29. P. Cullicover et L. McNally (eds). New York: Academic Press: 281-302.

Quirk, R. et al. 1972. A Grammar of Contemporary English. Londres: Longman.

Rizzi, L. 1997. The Fine Structure of the Left Periphery. In L. Haegeman (ed.), Elements of Grammar: Handbook of Generative Syntax. Dordrecht: Kluwer: 28I-337.

Ross, J.R. 1973. The Same Side Filter. In C. Corum, T. Smith-Stark et A. Weiser (eds), Papers from the Ninth Regional Meeting of the Chicago Linguistic Society. Chicago: University of Chicago: $549^{-567}$.

Tizón-Couto, D. 2009. A Corpus-based Analysis of Left Dislocation in Modern English. In D. Apotheloz et al. (eds), Les linguistiques du détachement. Actes du colloque international de Nancy, 7-9 juin 2006. Berne: Peter Lang: 551-565.

Ward, G.L. 1988. The Semantics and Pragmatics of Preposing. New York: Garland.

WARD, G.L. 2008. Seminar on information structure and word order variation: preposing. Accessible en ligne sur http://www.usc-vlcg.es/ppt\%2oward/preposing_revised.ppt (consulté le 20/OI/20IO). 